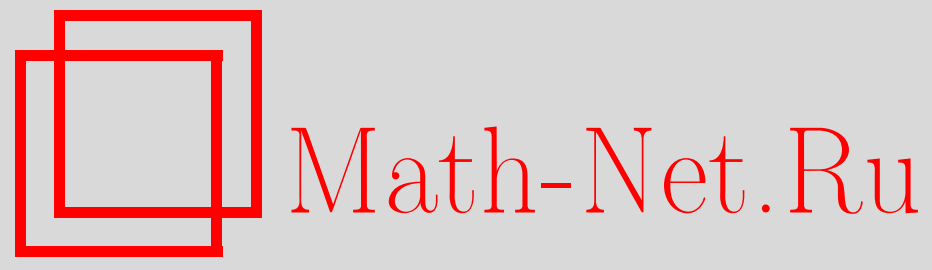

А. Ю. Самарин, Пространственная локализация квантовой частицы, Вестн. Сам. гос. техн. ун-та. Сер. Физ.-мат. науки, 2013, выпуск 1(), 387-397

DOI: https://doi.org/10.14498/vsgtu1138

Использование Общероссийского математического портала Math-Net.Ru подразумевает, что вы прочитали и согласны с пользовательским соглашением

http://www.mathnet.ru/rus/agreement

Параметры загрузки:

IP : 52.87 .193 .239

26 апреля 2023 г., 10:11:13

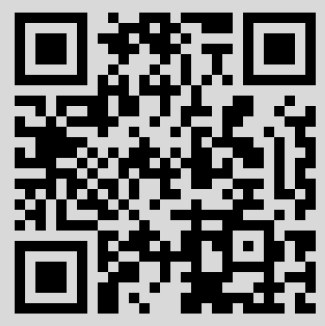


Вестн. Сам. гос. техн. ун-та. Сер. Физ.-мат. науки. 2013. № 1 (30). С. 387-397

УДК 53.03:(539.183-539.194)

\title{
ПРОСТРАНСТВЕННАЯ ЛОКАЛИЗАЦИЯ КВАНТОВОЙ ЧАСТИЦЫ
}

\section{А. Ю. Самарин}

Самарский государственный технический университет,

Россия, 443100, Самара, ул. Молодогвардейская, 244.

E-mail: samarinay@yahoo.com

\begin{abstract}
Показано, что наряду с эволюиионным механизмом изменения волновой функции квантовой частиць волновое уравнение в интегральном виде содержит также и математический механизм, позволяющий описать редукцию волновой функиии как физический процесс. Такое описание представлено для процесса пространственной локализации квантовой частищъ при измерении её координаты и показано, что в результате взаимодействия квантовой частиць со специфическим макроскопическим обгектом - измерителъным прибором возникает коллапс волновой функиии. Математический образ этого физического явления имеет вид мгновенной редукции множества виртуальных траекторий движения квантовой частиць к их подмножеству, определяемому условиями измерения, при возникновении макроскопических изменений в приборе. $B$ традиционной квантовой механике такой редукции должна соответствовать не эволючия вектора состояния в гилъбертовом пространстве, а коллапс самого гильбертова пространства.
\end{abstract}

Ключевые слова: коллапс волновой функиии, пространственно-временное описание пространственной локализачии, нелокальность процесса редукции.

Понятие редукции волновой функции в традиционной квантовой механике обозначает такое изменение волновой функции квантового объекта при его взаимодействии с измерительным прибором, которое не имеет непрерывной временной зависимости [1]. Тип волновой функции, возникающей в результате такого изменения, определяется редукцией двух видов: пространственной локализацией квантового объекта целиком или его части. Так, измерение координаты атома соответствует его пространственной локализации, тогда как измерение его энергии соответствует пространственной локализации атома измерительного инструмента (например, фотоплёнки), с которым объект измерения находится в запутанном состоянии. В любом случае физическая основа редукции обоих видов сводится к одному и тому же процессу - пространственной локализации квантового объекта [2]. Поэтому для выяснения её сущности достаточно рассмотреть процесс пространственной локализации квантовой частицы, подразумевая при этом, что квантовая частица представляет собой однородный объект (в смысле его целостности во всех квантово-механических процессах), имеющий пространственное распределение. Термин «частица» указывает на то, что такой объект при пространственной локализации преобразуется в единственную точку (в дальнейшем в качестве квантового объекта будет рассматриваться исключительно квантовая частица).

Редукция волновой функции, имеющая место при пространственной ло-

Алексей Юръевич Самарин (к.ф.-м.н), доцент, каф. общей физики и физики нефтегазового производства. 
кализации квантовой частицы, с общепринятой на сегодняшний день точки зрения представляет собой математическую процедуру, устанавливающую соответствие между волновой функцией координат квантовой частицы и единственным результатом измерения её координаты. Эта процедура не имеет в свой основе никакого конкретного физического процесса, который мог бы быть описан в терминах эволюции вектора состояния в гильбертовом пространстве. Публикации, посвященные описанию редукции [3], либо сводят её к чисто математическим процедурам [4], либо связывают её с процессами, находящимися за пределами описания квантовой механики [5,6]. Проблема, возможно, связана с тем обстоятельством, что физические процессы в традиционной квантовой механике описываются эволюцией вектора состояния в гильбертовом пространстве, тогда как состояние, возникающее в результате редукции, вообще не относится к исходному гильбертову пространству [7]. Другими словами, процесс редукции определяется скорее не эволюцией волновой функции как вектора состояния в гильбертовом пространстве, а преобразованием самого гильбертова пространства. В результате исходное гильбертово пространство преобразуется либо (в пределе) к двумерному пространству (по одному измерению для частицы и прибора) в случае редукции первого вида, либо в многообразие исходного гильбертова пространства - для второго вида редукции. Так или иначе, как физический процесс редукция выходит за рамки возможностей описания методами традиционной квантовой механики.

Для описания физического процесса пространственной локализации квантовой частицы при её взаимодействии с измерителем можно использовать интегральное волновое уравнение вида $[8,9]$

$$
\Psi_{t_{2}}\left(x_{2}\right)=\int K\left(x_{1}, x_{2}\right) \Psi_{t_{1}}\left(x_{1}\right) d x_{1},
$$

где $x_{1}, t_{1}, x_{2}, t_{2}$ - координаты пространства и моменты времени, соответствующие начальной и конечной волновым функциям (здесь и далее рассматривается только одномерное движение); $\Psi_{t_{1}}\left(x_{1}\right), \Psi_{t_{2}}\left(x_{2}\right)$ - волновые функции начального и конечного состояний; $K_{t_{1}, t_{2}}\left(x_{1}, x_{2}\right)$ - амплитуда перехода. Сам по себе математический объект, соответствующий традиционному для квантовой механики термину «амплитуда перехода», не предполагает наличия вероятности в описании, а в данном случае просто характеризует перемещение квантового объекта в физическом пространстве. Амплитуда записывается в форме интеграла по траекториям (интеграл по траекториям берётся в математически наиболее корректном виде континуального интеграла [7]):

$$
K_{t_{1}, t_{2}}\left(x_{1}, x_{2}\right)=\int \exp \frac{i}{\hbar} S_{1,2}[\gamma][d \gamma],
$$

где $S_{1,2}[\gamma]$ - классический функционал действия для виртуальной траектории $\gamma \equiv x(t)$, которая имеет начало в точке пространства $x_{1}$ и конец в точке $x_{2}$ в моменты времени $t_{1}$ и $t_{2}$ соответственно; $[d \gamma]$ - элемент объёма пространства виртуальных траекторий [7]. В процессе взаимодействия участвует классический прибор. Для выполнения функции, соответствующей цели настоящей работы - пространственной локализации квантовой частицы, он, вопервых, должен быть макроскопическим объектом, состоящим из пространственно локализованных частей, и, во-вторых, быть способным усиливать 
взаимодействие с квантовым объектом до макроскопического уровня [10]. По отношению к волновому уравнению (1) под макроскопическим уровнем понимается такое значение минимального функционала действия для всех виртуальных траекторий в (2), при котором вклад в континуальный интеграл, определяющий амплитуду перехода, дает только соответствующая траектория и бесконечно близкие к ней [9]. Другими словами, макроскопическому процессу соответствует единственная траектория движения системы в конфигурационном пространстве.

Рассмотрим замкнутую систему, состоящую из измерительного инструмента и квантовой частицы. Пусть измерительный инструмент состоит из $n$ активных элементов (например, зёрен фотопленки), имеющих достаточно малый для обеспечения точности определения координаты частицы, но макроскопический размер. Результатом взаимодействия квантовой частицы с активными элементами прибора является возникновение макроскопических изменений в одном из них. Предположим, что эти изменения возникают в результате макроскопического процесса $A^{j}$ (регистрирующего процесса), который инициируется в активном элементе $j$ взаимодействием с квантовой частицей. Пусть $X^{j}$ - обобщённая координата этого процесса. Она характеризует всю совокупность квантовых объектов, участвующих в нём (активных частиц измерителя). До возникновения регистрирующего процесса эти координаты $X^{j}$ определяют свойства активных частиц измерителя по отношению к возможности инициации регистрирующего процесса. Предположим, что взаимодействие между прибором и квантовой частицей до возникновения регистрирующего процесса полностью описывается координатами прибора $X^{j}$ и координатой квантовой частицы $x$. Другими словами, потенциальная энергия взаимодействия прибора и частицы-объекта зависит явно только от этих координат. Тогда квантовое взаимодействие частицы-объекта с прибором будет описываться запутанной волновой функцией $\Psi\left(x, X^{1}, \ldots, X^{n}\right)$, эволюция которой определяется интегральным волновым уравнением

$$
\begin{aligned}
\Psi_{t_{2}}\left(x_{2}, X_{2}^{1}, \ldots, X_{2}^{n}\right) & = \\
=\int \cdots \int K_{t_{2}, t_{1}}\left(x_{2},\right. & \left.x_{1}, X_{2}^{1}, \ldots, X_{2}^{n}, X_{1}^{1}, \ldots, X_{1}^{n}\right) \times \\
& \times \Psi_{t_{1}}\left(x_{1}, X_{1}^{1}, \ldots, X_{1}^{n}\right) d x_{1} d X_{1}^{1} \cdots d X_{1}^{n},
\end{aligned}
$$

где нижний индекс 1 обозначает начальные, нижний индекс 2 - текущие значения физических величин; верхний индекс указывает номер активного элемента измерителя; $K_{t_{2}, t_{1}}\left(x_{2}, x_{1}, X_{2}^{1}, \ldots, X_{2}^{n}, X_{1}^{1}, \ldots, X_{1}^{n}\right)$ - амплитуда перехода (все временные зависимости носят параметрический характер [1]). В соответствии с работой [9] для системы, имеющей $n+1$ степень свободы $(n$ степеней относятся к измерителю, 1 - к квантовой частице), амплитуда перехода может быть записана в виде интеграла по траекториям

$$
\begin{aligned}
K_{t_{2}, t_{1}}\left(x_{2}, x_{1}, X_{2}^{1}, \ldots, X_{2}^{n}, X_{1}^{1}, \ldots, X_{1}^{n}\right)= \\
\quad=\int \cdots \int \exp \frac{i}{\hbar} s_{1,2}[\gamma] \prod_{j=1}^{n} \exp \frac{i}{\hbar} S_{1,2}\left[\Gamma^{j}\right][d \gamma]\left[d \Gamma^{1}\right] \cdots\left[d \Gamma^{n}\right],
\end{aligned}
$$


где $\gamma$ - виртуальная траектория квантовой частицы (термин квантовая частица, как и ранее, не предполагает локализованный в пространстве объект, поэтому в такой интерпретации, термин виртуальная траектория квантовой частицы обозначает виртуальную траекторию индивидуальной точки её пространственного распределения); $\Gamma^{j} \equiv X^{j}(t)$ - виртуальная траектория, соответствующая измерительному процессу (квантовому процессу, возникающему в макроскопическом активном элементе $j$ в результате взаимодействия с квантовой частицей); $s_{1,2}[\gamma]$ - функционал действия для траектории квантового объекта (он зависит через потенциальную энергию и от координат измерительного процесса); $S_{1,2}\left[\Gamma^{j}\right]$ - функционал действия для траектории измерительного процесса (он имеет аналогичную зависимость от координат квантовой частицы). Эти функционалы связывают квантовую частицу и активные элементы измерителя в единую систему, описываемую запутанной волновой функцией.

Пусть в отсутствие взаимодействия активные частицы измерителя находятся в стационарном состоянии на дне потенциальной ямы, природа которой в данном случае не имеет значения. При движении этих частиц в её пределах потенциальная энергия, входящая в функционал действия $S_{1,2}\left[\Gamma^{j}\right]$, исчисляется относительно дна этой ямы. Предположим, что глубина этой потенциальной ямы имеет такой же порядок величины, как и значение энергии взаимодействия квантовой частицы-объекта с активными частицами измерителя (то есть взаимодействие с квантовой частицей может привести к "выходу"активных частиц измерителя из этой потенциальной ямы). Далее предположим, что активные частицы измерителя взаимодействуют и с другими частями измерителя, которые не принимают непосредственного участия во взаимодействии с квантовой частицей-объектом, причём соответствующая потенциальная энергия имеет макроскопическую величину. Другими словами, энергия, соответствующая дну описанной выше потенциальной ямы, имеет макроскопическое значение благодаря наличию такого взаимодействия (эта энергия является внутренней по отношению к измерителю в целом, но до момента инициации макроскопического процесса - внешней по отношению к системе, описываемой волновой функцией (3)). Возможность такой ситуации предполагает второе свойство измерителя - усиление квантового взаимодействия (примером энергии, обеспечивающей процесс усиления, может служить энергия электронов катода в поле фотоэлектронного умножителя, химическая энергия молекул фотоплёнки и т.п.).

Тогда при превышении значения энергии активными частицами измерителя, достаточного для выхода из потенциальной ямы (за счёт энергии взаимодействия с квантовой частицей), их потенциальная энергия мгновенно возрастает и возникает макроскопический $A$-процесс. Поскольку потенциальная энергия $A$-процесса имеет макроскопическое значение, для активного элемента, в котором он возникает, например $k$, амплитуда перехода будет полностью определяться действием $S_{1,2}\left[\Gamma_{A}^{k}\right]=-U_{A}^{k} t$ для единственной траектории $\Gamma_{A}^{k}$ классического процесса в интервале времени, когда $T \ll U$. Рассмотрим преобразование волновой функции системы (3) с момента времени $t_{3}$ непосредственно перед началом $A$-процесса до момента времени $t_{4}=t_{3}+\varepsilon$, когда функционалы действия для всех виртуальных траекторий становятся пренебрежимо малыми по сравнению с величиной $S_{1,2}\left[\Gamma_{A}^{k}\right]$. Так как величина 
потенциальной энергии, входящая в функционал действия для траектории макроскопического процесса, имеет макроскопическое значение, то промежуток времени $\varepsilon$ можно рассматривать как бесконечно малую величину.

Пусть радиус взаимодействия квантовой частицы с измерителем много меньше размеров макроскопических активных элементов. Тогда все множество виртуальных траекторий квантовой частицы можно разделить на два подмножества, не имеющих общих элементов. Подмножество $(I)$ составляют траектории, проходящие через область пространства $(I)$, находясь в которой квантовая частица взаимодействует с активным элементом $j=k$; подмножество $(I I)$ составляют все прочие виртуальные траектории (проходящие через область пространства $(I I)$, где взаимодействие с этим элементом отсутствует). Нормировка волновой функции может быть записана в виде

$$
\begin{aligned}
\int \cdots \int_{\Omega}\left|\Psi_{t_{4}}\left(x_{4}, X_{4}^{1}, \ldots, X_{4}^{n}\right)\right|^{2} d x_{4} d X_{4}^{1} \cdots d X_{4}^{n}= \\
=\int \cdots \int_{I}\left|\Psi_{t_{4}}\left(x_{4}, X_{4}^{1}, \ldots, X_{4}^{n}\right)\right|^{2} d x_{4} d X_{4}^{1} \cdots d X_{4}^{n}+ \\
\quad+\int \cdots \int_{I I}\left|\Psi_{t_{4}}\left(x_{4}, X_{4}^{1}, \ldots, X_{4}^{n}\right)\right|^{2} d x_{4} d X_{4}^{1} \cdots d X_{4}^{n}=1
\end{aligned}
$$

где $\Omega$ - область интегрирования, соответствующая как всем возможным значениям координат физического пространства квантового объекта, так и всем возможным значениям обобщённых координат измерительных процессов. Область интегрирования $I$ соответствует значениям переменных, при которых квантовая частица взаимодействует с $k$-тым прибором; $I I-$ прочим значениям переменных. Определить влияние возникновения макроскопического $A$ процесса на нормировку волновой функции позволяет математическая процедура, аналогичная представленной в [7]. Для её осуществления время следует представить в комплексном виде:

$$
t=\tau e^{i \varphi}
$$

где $\tau$ и $\varphi$ - вещественные величины (для реальной физической ситуаций $\varphi=$ $=0)$. Далее рассмотрим поворот в комплексной плоскости времени на угол $\frac{\pi}{2}$ против часовой стрелки. В этом случае функционалы действия примут вид

$$
i \int(T-U) d \tau
$$

где $T$ и $U$ - кинетическая и потенциальная энергии. При этом нормировка (5) преобразуется к виду

$$
\begin{gathered}
\int \cdots \int_{I}\left|\Psi_{\tau_{4}}\left(x_{4}, X_{4}^{1}, \ldots, X_{4}^{n}\right)\right|^{2} d x_{4} d X_{4}^{1} \cdots d X_{4}^{n}+ \\
+\int \cdots \int_{I I}\left|\Psi_{\tau_{4}}\left(x_{4}, X_{4}^{1}, \ldots, X_{4}^{n}\right)\right|^{2} d x_{4} d X_{4}^{1} \cdots d X_{4}^{n}= \\
\quad=\left.f(\varphi)\right|_{\varphi=\pi / 2}=\text { const }=C
\end{gathered}
$$


а амплитуда перехода (4) запишется так:

$$
\begin{aligned}
& K_{\tau_{2}, \tau_{1}}\left(x_{2}, x_{1}, X_{2}^{1}, \ldots, X_{2}^{n}, X_{1}^{1}, \ldots, X_{1}^{n}\right)= \\
& \quad=\int \cdots \int \exp \left(-\frac{1}{\hbar} s_{1,2}[\gamma]\right) \prod_{j=1}^{n} \exp \left(-\frac{1}{\hbar} S_{1,2}\left[\Gamma^{j}\right]\right)[d \gamma]\left[d \Gamma^{1}\right] \cdots\left[d \Gamma^{n}\right] .
\end{aligned}
$$

Малая величина времени $\varepsilon$ определяет большие значения кинетической энергии для переходов между областями пространства $I$ и $I I$, и их вкладом в амплитуду перехода можно пренебречь. Кроме того, на этом же основании можно пренебречь изменением положения квантовой частицы для виртуальных траекторий по сравнению с размером активных элементов измерителя, что сохраняет множества траекторий $I$ и $I I$ в неизменном виде в процессе коллапса. Эти два обстоятельства позволяют рассматривать эволюцию волновой функции в областях пространства $I$ и $I I$ независимо. Тогда, если учесть единственность макроскопической траектории $\Gamma_{A}^{k}$ и независимость соответствующего функционала действия от физических характеристик квантовой частицы, то волновая функция (3), определяемая амплитудой (7) для множества траекторий $I$, может быть переписана в виде

$$
\begin{aligned}
\Psi_{\tau_{4}}\left(x_{4}, X_{4}^{k}\right)= & \\
=\exp \left(\frac{1}{\hbar} U_{A}^{k} \varepsilon\right) \iint\left\{\int\right. & \left.\exp \left(-\frac{1}{\hbar} s_{3,4}\left[\gamma^{k}\right]\right)\left[d \gamma^{k}\right]\right\} \times \\
& \times \delta\left(X_{3}^{k}-X_{3, A}^{k}\right) \Psi_{\tau_{3}}\left(x_{3}, X_{3}^{k}\right) d x_{3} d X_{3}^{k},
\end{aligned}
$$

где $\gamma^{k}$ - виртуальные траектории квантовой частицы, проходящие через активный элемент $k$ во время коллапса; $X_{3 A}^{k}$ - обобщённая координата макроскопического процесса в момент времени $\tau_{3}$. Порядки величин слагаемых, входящих в нормировку (6), в момент времени $\tau_{4}$ определяются амплитудами перехода для интервала времени $i \varepsilon$. Первое слагаемое нормировки содержит квадрат экспоненциального множителя, в показатель которого, в соответствии с (8), входит макроскопическая потенциальная энергия $V_{A}^{k}$, тогда как второе слагаемое зависит исключительно от микроскопических значений потенциальных энергий. Благодаря этому обстоятельству нормировка (6) преобразуется к виду

$$
\int \cdots \int_{I}\left|\Psi_{\tau_{4}}\left(x_{4}, X_{4}^{1}, \ldots, X_{4}^{n}\right)\right|^{2} d x_{4} d X_{4}^{1} \cdots d X_{4}^{n}=C .
$$

Аналитическое продолжение этого выражения на вещественную ось времени дает аналогичную формулу для нормировки волновой функции для реального времени:

$$
\int \cdots \int_{I}\left|\Psi_{t_{4}}\left(x_{4}, X_{4}^{1}, \ldots, X_{4}^{n}\right)\right|^{2} d x_{4} d X_{4}^{1} \cdots d X_{4}^{n}=1 .
$$

Этой нормировке соответствует волновая функция 


$$
\begin{aligned}
\Psi_{t_{4}}\left(x_{4}, X_{4}^{k}\right) \exp \left(-\frac{i}{\hbar} U_{A}^{k} \varepsilon\right) \iint\left(\left\{\int\right.\right. & \left.\exp \frac{i}{\hbar} s_{3,4}\left[\gamma^{k}\right]\left[d \gamma^{k}\right]\right\} \times \\
& \left.\times \delta\left(X_{3}^{k}-X_{3, A}^{k}\right) \Psi_{t_{3}}\left(x_{3}, X_{3}^{k}\right)\right) d x_{3} d X_{3}^{k}
\end{aligned}
$$

где $\gamma^{k}$ - виртуальные траектории квантовой частицы, проходящие через активный элемент $k$ прибора в момент времени коллапса (принадлежащие множеству $I$ ). После возникновения макроскопического процесса исчезает зависимость траекторий измерительного процесса в $k$-том элементе от движения квантовой частицы. Эволюция квантовой частицы с этого момента времени определяется только траекториями $\gamma^{k}$, которые проходят через элемент $k$ в момент времени $t_{3}$. Благодаря этому запутанное состояние прибора и частицы распадается на два отдельных состояния. Если пренебречь пространственным размером активных элементов измерителя (для этого необходимо, чтобы изменение начальной волновой функции происходило на расстояниях, существенно превышающих этот размер), то пространственное положение элемента $k$ может быть определено одной координатой $Y^{k}\left(t_{4}\right) \approx Y^{k}\left(t_{3}\right)$. Тогда для бесконечно малого радиуса взаимодействия квантовой частицы и измерительного инструмента (что требуется при измерении координаты) волновая функция частицы непосредственно после измерения примет вид

$$
\Psi_{t_{4}}\left(x_{4}\right)=\delta\left(x_{4}-Y\left(t_{4}\right)\right) \approx \delta\left(x_{4}-Y\left(t_{3}\right)\right),
$$

где $t_{4}=t_{3}+\varepsilon-$ время окончания коллапса.

Тот факт, что интервал времени $\varepsilon$ может быть произвольно малым, а размер области пространства, в которой волновая функция системы до редукции отлична от нуля, может быть произвольно большим, не позволяет рассматривать процесс пространственной локализации как движение квантового объекта в соответствии с интегральным волновым уравнением из-за необходимости допущения произвольно больших значений скоростей движения. Скорее, коллапс волновой функции является проявлением единовременного изменения внутренней пространственной структуры квантового объекта. Таким образом, интегральное волновое уравнение (1), рассматриваемое в качестве постулата вместо уравнения Шредингера, описывает два возможных механизма изменения пространственных характеристик квантового объекта. Первый из них - эволюционный механизм - связан с изменением координатной волновой функции, аналогичным изменению функции плотности сплошной среды при её движении в пространстве (аналогия, конечно, не полная). Второй - редукционный механизм - предполагает наличие взаимодействия с макроскопическим объектом особого вида - измерительным прибором, при котором происходит изменение внутренней структуры микрообъекта, коллапс базиса его гильбертова пространства. Математически это выражается в мгновенном изменении множества возможных виртуальных траекторий движения объекта после инициации макроскопического процесса в измерителе.

Единственность пространственной точки локализации частицы обеспечивается тем обстоятельством, что взаимодействие между квантовой частицей и классическим прибором прекращается сразу после возникновения макроскопического процесса в одном из активных элементов измерителя. Физи- 
чески это позволяет рассматривать квантовую частицу как распределённый в пространстве объект, который проявляется в измерительном процессе как локализованная в пространстве точка.

В процессе измерения квантовая система, состоящая из квантовой частицы объекта и активных частиц измерителя, не является замкнутой (по отношению к оставшимся частям измерителя, обеспечивающим усиление процесса взаимодействия и регистрацию его результатов). В такой ситуации невозможно описать эволюцию системы, используя только переменные этой системы, относящиеся к исследуемому (текущему) моменту времени [11]. Чтобы исключить из описания переменные окружающей среды (оставшихся частей прибора), необходимо учитывать переменные системы, относящиеся к прошлым моментам времени. Тогда влияние окружающей среды на систему может быть учтено в виде временных зависимостей её потенциальной энергии, соответствующих различным траекториям переменных системы в прошлом (в настоящей работе была введена временная зависимость потенциальной энергии, имеющая скачок от микроскопического значения до макроскопического для траекторий, проходящих через один из активных элементов). Зависимость текущего состояния системы от переменных, относящихся к прошлому, содержится в фейнмановском представлении квантовой механики, которое в терминологии механики сплошной среды представляет собой квантовый аналог классического описания Лагранжа. То обстоятельство, что система не является замкнутой в процессе измерения, не позволяет осуществить его динамическое описание методом Эйлера, то есть в терминах эволюции вектора состояния в гильбертовом пространстве.

Фейнмановское описание квантовой эволюции не содержит наблюдаемых (в общем случае координаты виртуальных траекторий не являются измеряемыми координатами квантового объекта, а волновая функция не является вектором гильбертова пространства). Применительно к обсуждаемому вопросу это обстоятельство определяет то, что запутанное квантовое состояние системы, состоящей из прибора и квантового объекта, при таком подходе не может быть представлено в виде суперпозиции собственных состояний наблюдаемой. Для случая пространственной локализации это видно из различия мощностей счётного множества наблюдаемых координат системы и континуума - координат виртуальный траекторий. Исходя из этого можно заключить, что как разложение волновой функции по относительным состояниям Эверетта (отказ от постулата наблюдаемой в этой работе относится к процессу редукции, а не к наблюдаемой как к характеристике квантового объекта, имеющей классическую форму) [12], так и матрица плотности, соответствующая суперотбору, в теории декогеренции [13] не описывают текущее состояние системы при измерении, а представляют собой способ записи его возможных результатов. При этом принципиальное отсутствие детального динамического описания эволюции системы при измерении не позволяет определить причину реализации единственного результата измерения в рамках физической теории (в [14] в качестве такой причины рассматривается разум наблюдателя).

Детерминистическое интегральное волновое уравнение описывает редукцию волновой функции - стохастический процесс. Поскольку в процессе редукции принимают участие квантовый объект и измерительный прибор, лю- 
бой из них может являться причиной возникновения случайности результатов измерения. Если исходить из того положения, что вероятностное описание является результатом недостаточной информации об объекте (а именно этого требует буквальное понимание принципа причинности), то в качестве источника стохастичности при редукции волновой функции следует рассматривать классический измерительный прибор, огромное число степеней свободы которого не позволяет описать его динамически. Кроме того, интерпретация волновой функции как математического образа квантовой частицы, которая в реальности представляет собой распределённый в пространстве физический объект, не позволяет рассматривать квантовый объект в качестве источника стохастичности редукции. В данной работе случайность результата локализации проявляется в неопределённости номера активного элемента измерителя, в котором возникает регистрирующий процесс. Эта неопределённость при заданной волновой функции квантовой частицы вызвана статистическим разбросом макроскопических характеристик активных элементов (таких как чувствительность по отношению к взаимодействию с квантовой частицей, от которой зависит потенциальная энергия взаимодействия, энергия инициации регистрирующего процесса). По сути, эти характеристики являются скрытыми параметрами, статистический разброс которых определяет случайный характер локализации. Однако, если статистические свойства измерителя не зависят от координат пространства (что, как правило, имеет место на практике), то пространственное распределение плотности вероятности локализации определяется волновой функцией квантовой частицы [15] (в случае наличия такой зависимости она может быть учтена и исключена из рассмотрения). Таким образом, благодаря классическому прибору возникает элемент случайности при проявлении пространственного распределения меры, задаваемой правилом Борна, в результате чего она проявляется как вероятность.

Если квантовый объект представляет собой совокупность взаимодействующих квантовых частиц, то в случае локализации одной из них в процессе измерения запутанная волновая функция всего объекта одновременно изменяется во всем пространстве. Запутанное состояние совокупности квантовых частиц характеризуется виртуальными траекториями всей совокупности этих частиц (в случае одномерного движения каждой из них виртуальные траектории представляют собой траектории в конфигурационном пространстве с размерностью, равной числу частиц). При локализации одной из частиц в интегральном волновом уравнении сохраняются только те виртуальные траектории, которые проходят через область локализации этой частицы в момент времени возникновения регистрирующего процесса. Такая редукция множества траекторий определяет влияние локализации одной из частиц на состояние нелокализованной части квантового объекта. Это влияние проявляется в изменении пространственного распределения меры, определяющей вероятности локализации нелокализованных частиц системы. Таким образом, предлагаемый подход объясняет нелокальную корреляцию вероятностей локализации квантовых частиц, находящихся в запутанных состояниях, даже при отсутствии «силового» взаимодействия между ними в момент времени локализации (разумеется, для возникновения запутанного состояния такое взаимодействие должно было существовать в прошлом). 


\section{БИБЛИОГРАФИЧЕСКИЙ СПИСОК}

1. И. фон Нейман, Математические основы квантовой механики. М.: Наука, 1964. 367 с. [J. von Neumann, Mathematical foundations of quantum mechanics. Moscow: Nauka, 1964. 367 pp.]

2. R. Penrose, The road to reality. A complete guide to the laws of the universe. New York: Alfred A. Knopf, Inc., 2005. xxviii+1099 pp.

3. A. Bassi, G. C. Girardi, "Dynamical reduction models" // Phys. Rep., 2003. Vol. 379, no. 56. Pp. 257-426.

4. G. C. Ghirardi, A. Rimini, T. Weber, "Unified dynamics for microscopic and macroscopic systems" // Phys. Rev. D, 1986. Vol. 34, no. 2. Pp. 470-491.

5. D. Bohm, B. J. Hiley, "Nonlocality and locality in the stochastic interpretation of quantum mechanics" // Phys. Rep., 1989. Vol. 172, no. 3. Pp. 93-122.

6. D. Deutsch, "Quantum theory as a universal physical theory" // Int. J. Theoret. Phys., 1985. Vol. 24, no. 1. Pp. 1-41.

7. J. Zinn Justin, Path Integrals in Quantum Mechanics. Oxford: Oxford University Press, 2004. 320+xiv pp.; русск. пер.: Ж. Зинн-Жюстен, Континуальный интеграл в квантовой механике. М.: Физматлит, 2006. 360 с.

8. R. P. Feynman, "Space-time approach to non-relativistic quantum mechanics"// Rev. Modern Physics, 1948. Vol. 20. Pp. 367-387.

9. R. P. Feynman, A. R. Hibbs, Quantum Mechanics and Path Integrals. New York: McGrawHill, 1965. 371+xii pp.; русск. пер: Р. Фейнман, А. Хибс, Квантовая механика и интегралы по траекториям. М.: Мир, 1968. 382 с.

10. А. Ю. Самарин, "Естественное пространство микрообъекта" // Вестн. Сам. гос. техн. ун-та. Сер. Физ.-мат. науки, 2011. №3(24). С. 117-128. [A. Yu. Samarin, "Natural space of the micro-object" // Vestn. Samar. Gos. Tekhn. Univ. Ser. Fiz.-Mat. Nauki, 2011. no. 3(24). Pp. 117-128].

11. R. P. Feynman, The Development of the Space-Time View of Quantum Electrodynamics / Nobel Lecture, December 11, 1965. Preprint les Prix Nobel en 1965. Stockholm: The Nobel Foundation, 1966; русск. пер.: Р. Фейнман, "Развитие пространственно-временной трактовки квантовой электродинамики" // УФН, 1967. Т. 91, № 1. С. 29-48.

12. H. Everett, III, "Relative state" formulation of quantum mechanics"// Rev. Mod. Phys., 1957. Vol. 29, no. 3. Pp. 454-462.

13. W. H. Zurek, "Environment-induced superselection rules"// Phys. Rev. D., 1982. Vol. 26, no. 8. Pp. $1862-1880$.

14. М. Б. Менский, "Квантовые измерения, феномен жизни и стрела времени: связи между "тремя великими проблемами" (по терминологии В. Л. Гинзбурга)" // УФН, 2007. Т. 177, № 4. C. 415-425; англ. пер.: M. B. Menskii, "Quantum measurements, the phenomenon of life, and time arrow: three great problems of physics (in Ginzburg's terminology) and their interrelation" // Phys. Usp., 2007. Vol. 50, no. 4. Pp. 397-407.

15. А. Ю. Самарин, "Механизм возникновения стохастичности в квантовой механике" // Вестн. Сам. гос. техн. ун-та. Сер. Физ.-мат. науки, 2012. №4(29). С. 188198. [A. Yu. Samarin, "The mechanism of the appearance of stochasticity in quantum mechanics" // Vestn. Samar. Gos. Tekhn. Univ. Ser. Fiz.-Mat. Nauki, 2012. no. 4(29). Pp. 188-198]. 


\section{MSC: 81S40, 58D30}

\section{SPACE LOCALIZATION OF THE QUANTUM PARTICLE}

\section{A. Yu. Samarin}

Samara State Technical University, 244, Molodogvardeyskaya st., Samara, 443100, Russia.

E-mail: samarinay@yahoo.com

It is shown, that in addition to the evolution quantum object motion wave equation in the integral form can describe the wave function reduction as a physical process. Such description is represented for the space localization process, taking place when the space coordinate is measured, and it is shown, that collapse arises, as the result of the quantum particle and corresponding measuring instrument interaction. This physical phenomenon mathematical image looks like the instantaneous transformation of the virtual paths set to the subset, determined by the measuring process conditions, when the macroscopic changes appears in the measuring instrument.In conventional quantum mechanics such Hilbert space collapse itself corresponds to such reduction phenomenon.

Key words: wave function collapse, space-time description of the space localization, reduction process nonlocal property.

Original article submitted $09 / \mathrm{X} / 2012$; revision submitted 13/II/2013.

Alexey Yu. Samarin (Ph. D. (Phys. \& Math.)), Associate Professor, Dept. of General Physics and Physics of Oil and Gas Production. 